\title{
Non-Dimensional Approach for Static Balancing of Rotational Flexures
}

\author{
Ezekiel G. Merriam \\ Brigham Young University - Provo \\ Larry L. Howell \\ Brigham Young University - Provo, lhowell@byu.edu
}

Follow this and additional works at: https://scholarsarchive.byu.edu/facpub

Part of the Mechanical Engineering Commons

\section{Original Publication Citation}

Merriam, E.G. and Howell, L.L., "Non-Dimensional Approach for Static Balancing of Rotational Flexures," Mechanism and Machine Theory, Vol. 84, doi:10.1016/ j.mechmachtheory.2014.10.006, pp. 90-98, 2015.

\section{BYU ScholarsArchive Citation}

Merriam, Ezekiel G. and Howell, Larry L., "Non-Dimensional Approach for Static Balancing of Rotational Flexures" (2015). Faculty Publications. 1553.

https://scholarsarchive.byu.edu/facpub/1553

This Working Paper is brought to you for free and open access by BYU ScholarsArchive. It has been accepted for inclusion in Faculty Publications by an authorized administrator of BYU ScholarsArchive. For more information, please contact ellen_amatangelo@byu.edu. 


\title{
Non-Dimensional Approach for Static Balancing of Rotational Flexures
}

\author{
Ezekiel G Merriam, Larry L Howell* \\ Brigham Young University \\ Department of Mechanical Engineering \\ 435 CTB, Provo UT 84602
}

\begin{abstract}
This work presents a nondimensional method for statically balancing flexural hinges, including those with stiffness that varies with load. Using a set of nondimensional parameters, it is shown that one can quickly design a balancing mechanism for an idealized hinge/torsion spring system. This method is then extended to load-dependent systems, and is demonstrated with the design of a balanced cross-axis-flexural pivot with stiffness that varies as a function of compressive preload. A physical prototype is built and tested to verify the design method. The prototype demonstrates an average stiffness reduction of $87 \%$ over an 80 degree deflection range. The method enables improved static balancing for systems where the balancing pre-load influences the systems forcedeflection behavior.
\end{abstract}

Keywords: compliant, flexure, cross-axis-flexural pivot, static balancing

\section{Background}

A compliant mechanism obtains its motion from the deflection of its constituent members. Because this eliminates sliding contact of surfaces, friction and subsequent wear can be avoided, leading to higher performance [1. Be5 cause of the strain energy associated with bending the flexible members, compli-

\footnotetext{
* Corresponding author: Phone 1-801-422-8037, Fax 1-801-422-0516

Email address: lhowell@byu.edu (Larry L Howell)
}

Preprint submitted to Mechanism and Machine Theory

May 29, 2014 
ant mechanisms generally have higher actuation effort compared to traditional mechanisms 22. Static balancing is one strategy for reducing the actuation effort of compliant mechanisms. However, the pre-load applied in statically balanced systems is often large enough to affect the stiffness of the system to be balanced. This deviation from the expected stiffness reduces the balancing effect. The objective of this research is to develop a non-dimensional approach for static balancing of compliant hinges that is generalized to systems with load-dependent stiffness.

Static balancing is often accomplished by adding auxiliary springs that provide energy storage [3]. As the mechanism is actuated, energy stored in the balancing elements is transfered to the deflected mechanism [4. This means that less energy must be added during actuation, thus reducing actuation effort [5]. This strategy has been effectively incorporated into applications such as the design of surgical instruments and prosthetics [4, 6, 7.

Balancing elements commonly incorporate a negative stiffness mechanism, such as buckled beams in linear systems, or preloaded linear springs in rotational systems [8]. Other approaches use gravity balancing or systems of ideal springs [4, 9, 10].

Design of statically balanced systems generally requires the use of optimiza${ }_{25}$ tion routines [8, 11, 12]. Usually, the optimization problem minimizes the change in a mechanism's stored energy or searches for an appropriate negative-stiffness mechanism [13]. Depending on the system under consideration, this optimization may incorporate finite element analysis (FEA) and topology optimization. This means that to design a statically balanced compliant mechanism, significant resources must be available to develop and validate the model being used. Additionally, optimization routines utilizing FEA can quickly become cumbersome due to the relatively long solution time of non-linear FEA and the many function calls of most optimization routines.

Finally, building practical statically balanced mechanisms is difficult because 35 the balancing element is often bulky, making the system much larger than is convenient [10]. 
This paper describes the development of a non-dimensional approach for static balancing for idealized systems and load-dependent systems. The next section introduces nomenclature used in the method development that follows.

40 A non-dimensional approach for idealized systems is discussed first, followed by a generalization to load-dependent systems. An example load-dependent system is designed, and prototype hardware is built and tested.

\section{Nomenclature}

In this work, "load-independent (LI) joint" is a joint with a rotational stiffness that is not a function of applied lateral loads. This is modeled as a pin joint with a torsional spring. A "load-dependent (LD) joint" is a joint whose stiffness changes when a lateral load is applied. An LD joint can be modeled as an LI joint if a relationship can be found between the applied lateral loads and joint stiffness.

In this work the statically balanced system consists of an LI joint of finite, constant stiffness that is balanced by the addition of a pre-loaded constantstiffness linear spring. The spring connects at points equidistant from the pivot, as shown in Figure 1. This simplified system can represent load-dependent systems with proper application of the pseudo-rigid-body model [1.

Variables and their relationships are included in the following lists. The first list is for variables directly related to balancing of LI compliant hinges, illustrated in Figure 1

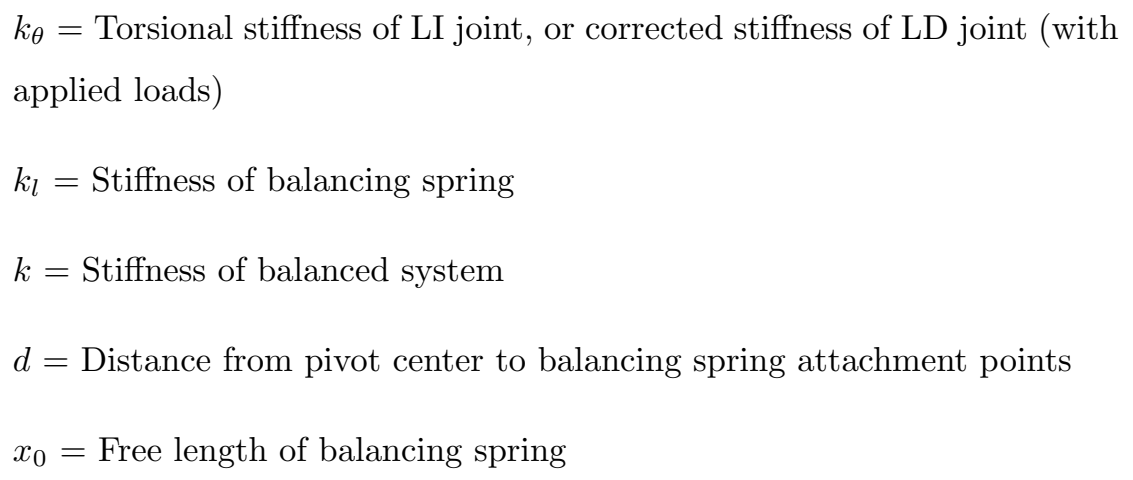




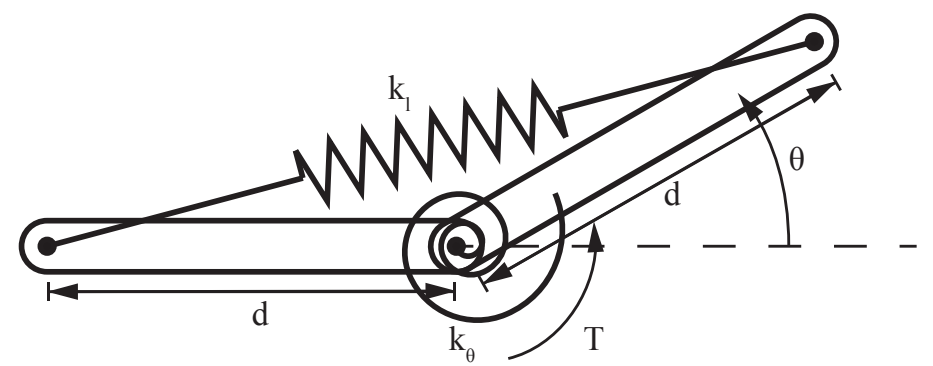

Figure 1: A LI system with balancing spring and associated variables.
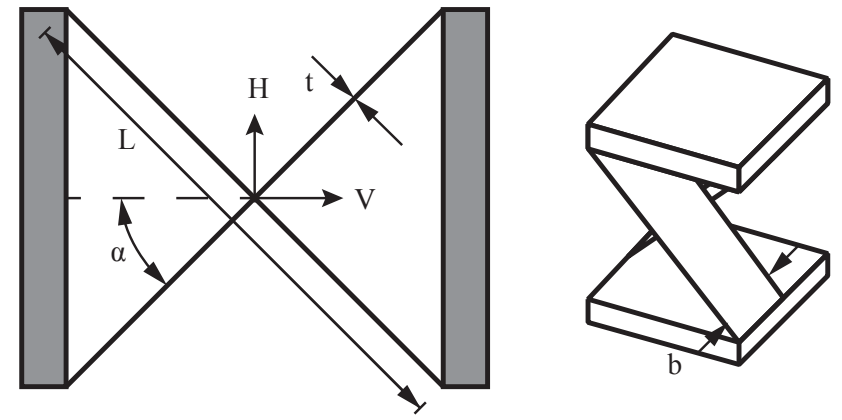

Figure 2: A cross-axis-flexural pivot with associated variables.

$P=k_{l}\left(2 d-x_{0}\right)=$ Preload applied to balancing spring

65

$\theta=$ Angle of deflection of the LI or LD joint

$T=k \theta=$ Torque required to deflect hinge through angle $\theta$

$\Pi_{1}=k_{\theta} /(P d)=$ Pi group governing torsional stiffness

$\Pi_{2}=k_{l} d / P=$ Pi group governing stiffness of balancing spring

The following list contains variables related to the design of a cross-axis-

70 flexural pivot (CAFP) that has a stiffness that is load-dependent. See Figure 2 for a depiction of geometric variables.

$E=$ Young's modulus of the flexure material

$b=$ Width of CAFP flexure strip 
$t=$ Thickness of CAFP flexure strip

$I=b t^{3} / 12=$ Moment of inertia of CAFP flexure strip

$L=$ Length of CAFP flexure strips

$k_{\theta}^{\prime}=$ Uncorrected torsional stiffness of LD joint (no applied loads)

The following list contains variables used to correct the stiffness of a CAFP to account for the effects of applied loads, as adapted from Wittrick [14. See

so Figure 2 for a depiction of geometric variables. The loads $V$ and $H$ are applied to the moving block of the CAFP at the center of the pivot.

$V=$ Vertical load applied to hinge

$H=$ Horizontal load applied to hinge

$\alpha=$ Half the intersection angle of the CAFP flexures

85

$v=V L^{2} \sec (\alpha) /(E I)=$ Non-dimensionalized applied vertical load

$h=H L^{2} \csc (\alpha) /(E I)=$ Non-dimensionalized applied horizontal load

$\beta_{i}=$ Dimensionless parameter describing the forces in CAFP flexures

$\phi_{i}=$ Dimensionless parameter describing the stiffness of the individual

CAFP flexures

90 3. Balancing of Load-Independent Hinge-Spring System

Because a general solution to static balancing is sought, it is desirable to use dimensional analysis techniques to analyze the balanced systems.

Recall the Buckingham-Pi theorem:

If an equation involving $k$ variables is dimensionally homogeneous, it can be reduced to a relationship among $k-r$ independent dimensionless products, where $r$ is the minimum number of reference dimensions required to describe the variables [15]. 
The energy of a load-independent system, $E$, can be written as the sum of the potential energies of the torsional and linear springs, as follows:

$$
E=\frac{k_{\theta}}{2} \theta^{2}+\frac{k_{l}}{2}\left(\sqrt{2 d^{2}(1+\cos (\theta))}-x_{0}\right)^{2}
$$

100 derivative with respect to $\theta$ as

$$
T=k_{\theta} \theta-\frac{k_{l}\left(\sqrt{2 d^{2}(1+\cos (\theta))}-x_{0}\right) d^{2} \sin (\theta)}{\sqrt{2 d^{2}(1+\cos (\theta))}} .
$$

Dividing by $\theta$ gives the mechanism stiffness $\left(k=\frac{T}{\theta}\right)$ as

$$
k=\frac{k_{\theta} \theta-\frac{k_{l}\left(\sqrt{2 d^{2}(1+\cos (\theta))}-x_{0}\right) d^{2} \sin (\theta)}{\sqrt{2 d^{2}(1+\cos (\theta))}}}{\theta} .
$$

This result will be used later. Setting the torque from Equation (2) equal to zero and using $x_{0}=2 d-P / k_{l}$ gives:

$$
k_{\theta} \theta=\frac{k_{l}\left(\sqrt{2 d^{2}(1+\cos (\theta))}-\left(2 d-P / k_{l}\right)\right) d^{2} \sin (\theta)}{\sqrt{2 d^{2}(1+\cos (\theta))}}
$$

105 $k_{\theta}, k_{l}, P$, and $d$ and two dimensions (force and length). Thus, $k=4$ and $r=2$, and the system can be described by two non-dimensional parameters, designated $\Pi_{1}$ and $\Pi_{2}$. Since it is desirable that these parameters have a physical, intuitive meaning it is convenient to select force $P$ and distance $d$ as repeat-

$$
\Pi_{1}=\frac{k_{\theta}}{P d}
$$

and

$$
\Pi_{2}=\frac{k_{l} d}{P}
$$


We now assume that a relationship between our pi-groups exists, written as $\Pi_{1}=\phi\left(\Pi_{2}\right)$. As a result of the Buckingham-Pi theorem, this relationship will govern the stiffness of the system. If a relationship for $\Pi_{1}$ and $\phi\left(\Pi_{2}\right)$ can be found that results in a balanced system, a combination of system parameters $k_{\theta}, k_{l}, P$, and $d$ that follows this relationship will yield a statically balanced system.

A program was written to find the relationship between $\Pi_{1}$ and $\Pi_{2}$ for $0.2 \leq$ $\Pi_{1} \leq 1$. This script used a particle swarm optimization [16 routine to minimize $\left|\frac{k}{k_{\theta}}\right|$ (see Equation (3)) calculated over a range of $0<\theta \leq 20^{\circ}$ for a given value of $\Pi_{1}$. Optimization variables were $k_{l}, P$, and $d . k_{\theta}$ was found from Equation (5). After minimizing the normalized stiffness $\left|\frac{k}{k_{\theta}}\right|, \Pi_{2}$ was calculated from Equation (6) using the final variable values. A particle swarm algorithm was employed for this optimization because of its ability to find a global optimum.

This approach was repeated for other values of $\Pi_{1}$ in the range of $0.2 \leq$ $\Pi_{1} \leq 1$ to find $\Pi_{2}$ as a function of $\Pi_{1}$. Figure 4 plots $\left|\frac{k}{k_{\theta}}\right|$ against $\Pi_{1}$. The plot shows that in the region of $\Pi_{1}=0.5$ a close approximation of perfect balancing is achieved. By plotting $\Pi_{2}$ as a function of $\Pi_{1}$, as in Figure 3, we can see that using $\Pi_{1} \geq 0.5$ gives $\Pi_{2}<0$, which is inconvenient for design purposes (it could require $k_{l}$ to be negative). Table 1 lists convenient $\Pi$ terms along with the expected reduction in stiffness. A curve fit for the data in Figure 3 is

$$
\Pi_{2}=-102.54 \Pi_{1}+51.104: 0.2 \leq \Pi_{1} \leq 0.81
$$

with an $R^{2}$ value of 1 .

Rearranging the data in Figure 4 and applying a curve fit gives the $\%$ reduction in stiffness as

$$
\frac{k_{\theta}-k}{k_{\theta}} \times 100= \begin{cases}2414 \Pi_{1}^{3}-3336 \Pi_{1}^{2}+1679 \Pi_{1}-206.7 & : 0.2 \leq \Pi_{1} \leq 0.5 \\ 111.85 \Pi_{1}^{2}-215.42 \Pi_{1}+179.53 & : 0.5 \leq \Pi_{1} \leq 0.8\end{cases}
$$

with an $\mathrm{R}^{2}$ value of 0.9998 . 


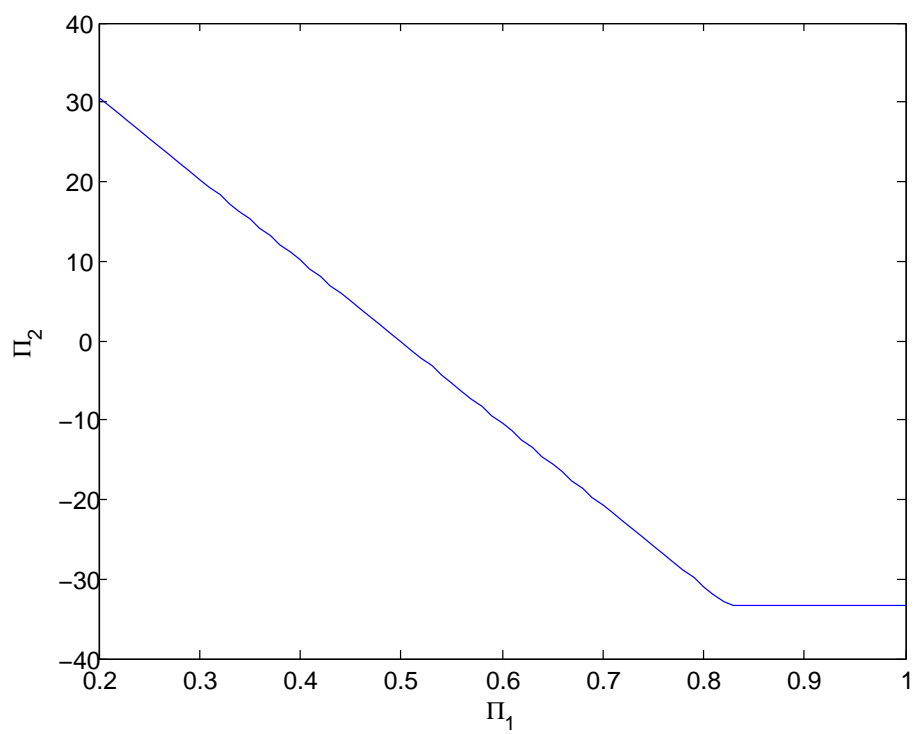

Figure 3: $\Pi_{2}$ plotted as a function of $\Pi_{1}$. The flat line occurring at $\Pi_{1}=0.82$ is an artifact of the optimization constraints.

This method only works when the flexure is load-independent; that is, when $k_{\theta}$ is not a function of applied lateral loads. Also, because the joint design has only four parameters $\left(k_{\theta}, k_{l}, P\right.$, and $\left.d\right)$, choosing more than two parameters results in an over-constrained design.

\section{Extending to Load-Dependent Joints}

In load-dependent (LD) systems, torsional stiffness varies with applied loading. Because the balancing spring exerts a pre-load on the flexure to be balanced, its stiffness $\left(k_{\theta}\right)$ is no longer the same as its stiffness without any applied loads $\left(k_{\theta}^{\prime}\right)$. To use the $\Pi$ groups with a LD joint, a prediction of joint stiffness under load is required. Once the corrected stiffness $k_{\theta}$ is found for a specified pre-load $P$, the other joint parameters can be found from $\Pi_{1}$ and $\Pi_{2}$.

In this work, we will consider the static balancing of a cross-axis-flexural pivot, sometimes called a cross-spring pivot. This is a type of flexure formed by crossing two flexible strips and has been used extensively to allow motion 


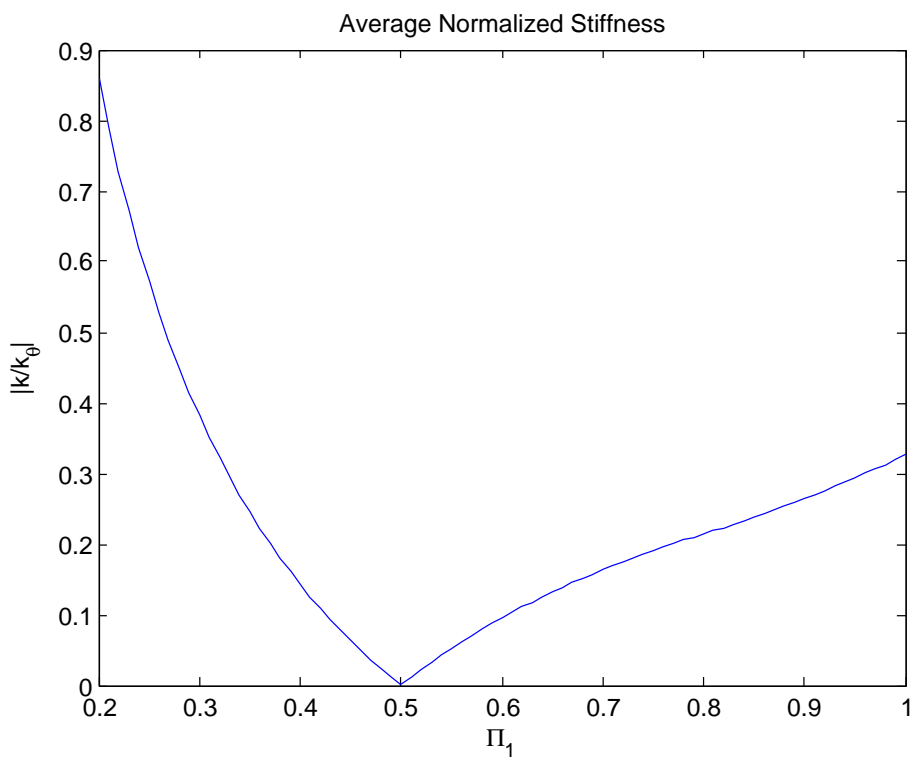

Figure 4: Normalized balanced stiffness as a function of $\Pi_{1}$

in many applications [14, 17, 18, 19, 20. Additionally, it has been the subject of other investigations into static balancing strategies [13. Morsch and Herder were able to balance a CAFP using a pair of zero-free-length springs with average stiffness reduction of $70 \%$ in the physical prototype [13]. A final motivation for the use of a CAFP is the availability of published load-dependent behavior [14, 18. In this work we use a different balancer topology and employ the methods

Table 1: Tabulated values of $\Pi_{1}$ and $\Pi_{2}$, with the expected reduction in joint stiffness.

\begin{tabular}{lcr}
$\Pi_{1}$ & $\Pi_{2}$ & $\frac{k_{\theta}-k}{k_{\theta}} \times 100$ \\
\hline \hline 0.5 & -0.1673 & 100.0 \\
0.49 & 0.8581 & 98.8 \\
0.48 & 1.884 & 97.6 \\
0.47 & 2.909 & 96.3 \\
0.46 & 3.934 & 95.0 \\
0.45 & 4.96 & 93.6 \\
\hline
\end{tabular}


described here to take into account the change in CAFP stiffness when subjected to a compressive load.

Wittrick established that the stiffness of cross-axis-flexural pivots is dependent on applied lateral loads [14, 18. He discussed how applied loads change the moments and loads applied to the constituent flexures, which affects their deflections. This same principle applies to many flexure systems commonly in use. A balancing method that accounts for the change in stiffness due to applied loads can provide a more balanced system. In this case, the applied load is due to the compressive pre-load of the balancing spring.

Wittrick's results are summarized here for convenience. He gives the stiffness of a CAFP as [14:

$$
k_{\theta}=\frac{E I}{L}\left(\phi_{1}+\phi_{2}\right)
$$

where

$$
\begin{gathered}
\phi_{i}=\beta_{i}\left(\cot \beta_{i}-\beta_{i}\right) \\
\beta_{1}^{2}=\frac{1}{8}(v+h) \\
\beta_{2}^{2}=\frac{1}{8}(v-h)
\end{gathered}
$$

Recall that $v$ and $h$ are non-dimensionalized horizontal and vertical loads. $P$. Choosing an acceptable value of $P$ and letting $V=-P$ and $H=0$, allows the computation of $k_{\theta}$ for a given geometry. Choosing a value for $\Pi_{1}$ and its associated $\Pi_{2}$ for the desired stiffness reduction enables the calulation of the required $d$ and $k_{l}$ from Equations (5) and (6). Thus the $\Pi$ groups reduce the balancing problem to a system of two equations and four unknowns. Choosing two unknowns as design parameters allows the equations to be solved.

Alternatively, if it is desirable to select a value of $P$ and $k_{l}$ with flexures of a given moment of inertia, the associated $k_{\theta}$ can be found to satisfy Equation (5), and $L$ can be found with an optimization loop. Because Equations (9) and (4) 
contain trigonometric terms, a non-gradient based optimization routine such as particle swarm optimization is effective.

\section{Example Design}

This approach was followed to design a statically balanced CAFP. Because the spring preload changes the behavior of the CAFP, implementing the loaddependent method introduced here can result in a system with lower stiffness than would otherwise be possible. Convenient values of $k_{l}$ and $P$ were chosen to match those of a commercially available tension spring, and a flexure moment of inertia was selected so that the CAFP could be built from available spring steel. A flexure length was found along with torsional stiffness $k_{\theta}$ and $d$. The resulting design variables are listed in Table 2

\begin{tabular}{ccr}
\multicolumn{3}{c}{ Table 2: Design parameters for balanced CAFP. } \\
Parameter & Value & Units \\
\hline \hline$\Pi_{1}$ & 0.49 & \\
$\Pi_{2}$ & 0.8581 & \\
$E$ & $30.0 \mathrm{e} 6$ & $\mathrm{lbs} / \mathrm{in}^{2}$ \\
$L$ & 2.5961 & in \\
$b$ & 0.015 & in \\
$w$ & 0.501 & in \\
$I$ & $1.409 \mathrm{e}-7$ & in \\
$k_{\theta}$ & 5.7054 & $\mathrm{lb}-\mathrm{in} / \mathrm{rad}$ \\
$k_{l}$ & 1.3 & $\mathrm{lb} / \mathrm{in}$ \\
$P$ & 4.2 & $\mathrm{lb}$ \\
$d$ & 2.7723 & in \\
$x_{0}$ & 2.3139 & in \\
\hline
\end{tabular}

This CAFP was fabricated and tested, as described in the following section. 


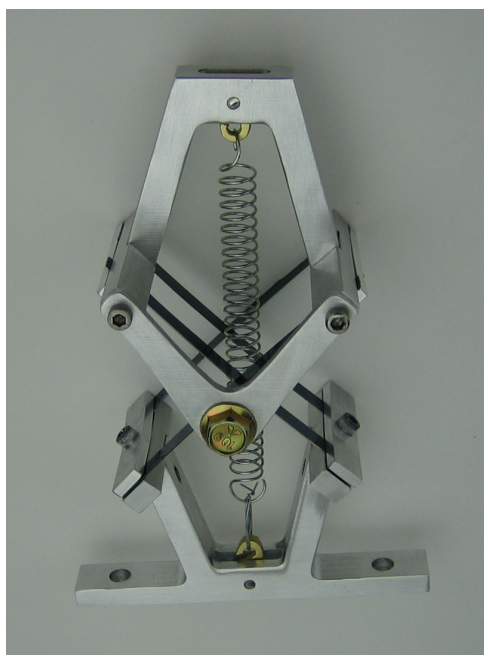

Figure 5: A prototype joint designed using the method detailed in this paper.

\section{Experimental Results and Discussion}

The prototype balanced joint was designed and fabricated according to the design parameters of Table 2. The final hardware is shown in Figure 5. Rigid sections were machined from 6061 aluminum bar stock while the flexures were cut from spring steel. Torque was measured using a torque transducer while the joint was displaced with a worm-wheel gear-set. The experimental setup is shown in Figure 6 .

Figure 7 shows the predicted and measured stiffness in both the unbalanced and balanced configurations. The balanced stiffness is not as low as predicted; this is most likely due to variance in the linear spring stiffness from nominal, the difficulty in ensuring precise application of the design pre-load $P$, as well as manufacturing error. The finite element results shown were obtained from an ANSYS simulation that used BEAM23 elements for the flexures and COMBIN14 elements for the linear spring. Simulations showed a stress-limited deflection of about $40^{\circ}$, so the prototype was designed with this physical limit in mind.

Figure 8 shows the percent stiffness reduction calculated as $\frac{k_{\theta}^{\prime}-k}{k_{\theta}^{\prime}} \times 100$. An average stiffness reduction of $87 \%$ was achieved over $80^{\circ}$ of deflection. Note that 


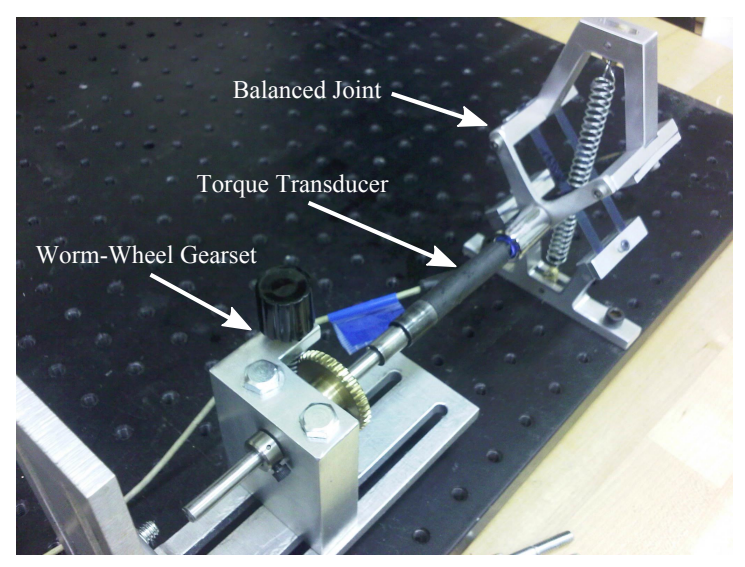

Figure 6: Experimental setup used to measure unbalanced and balanced stiffness.

stiffness reduction in Table 1 is calculated as $\left|\left(k_{\theta}-k\right) / k_{\theta}\right|$, while the stiffness reduction shown in Figure 8 is $\left|\left(k_{\theta}^{\prime}-k\right) / k_{\theta}^{\prime}\right|$, and the compressive load $P$ makes $k_{\theta}^{\prime}<k_{\theta}$.

This prototype demonstrates the validity of the balancing method presented herein. Using non-dimensional parameters as a balancing criterion simplifies the design process, making the rapid design of balanced joints practical in many applications. By taking into account the change in stiffness of a flexure due to joint pre-load, a better balancing solution can be achieved than if the flexure stiffness is assumed to be independent of load.

\section{Conclusion}

It has been shown that the use of the $\Pi$ groups presented herein can simplify the design of balancing mechanisms for compliant hinges that exhibit loadindependent behavior. It has also been shown that the $\Pi$ groups are equally valid when used in conjunction with load-dependent joints whose stiffness under load can be predicted.

A prototype CAFP was built and tested. Results show that the stiffnesscorrection method results in highly balanced joints with an $87 \%$ average stiffness reduction over an $80^{\circ}$ deflection range. The method enables balancing results 


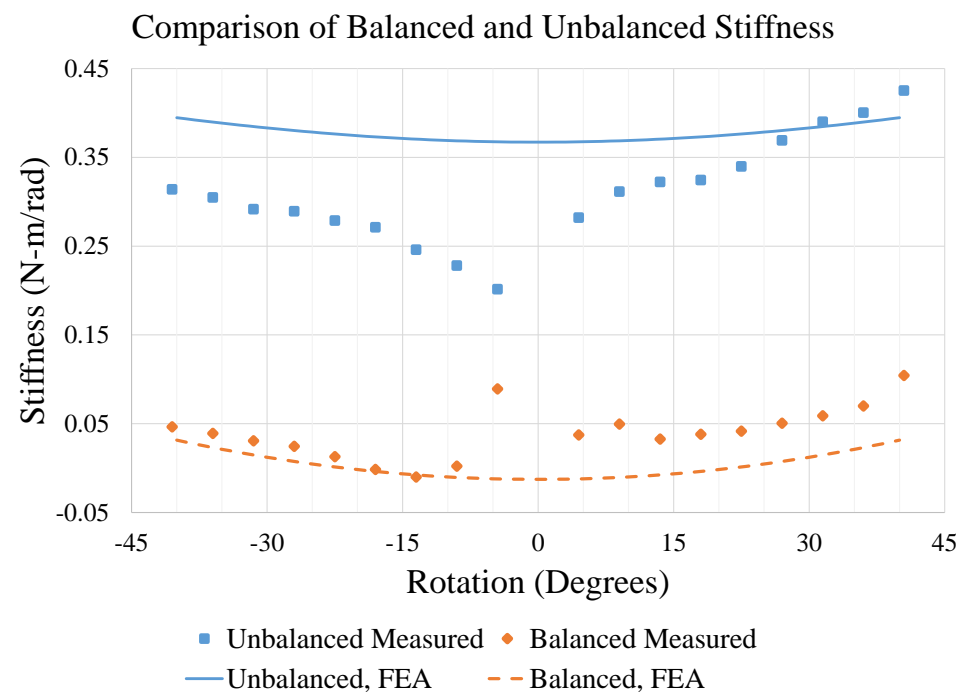

Figure 7: The stiffness of the balanced and unbalanced joints plotted for comparison.

that would not be possible without considering the effect that the balancing preload has on the system stiffness. The balancing method and stiffness correction were demonstrated with a cross-axis-flexural pivot, but the result is general and can be applied to joints having load-dependent stiffness.

\section{Acknowledgments}

The author express thanks to Abraham Lee for providing the particle swarm optimization routine, to Ken Forster for assistance in fabricating the CAFP prototype, and Kevin Cole for his help setting up the data acquisition system. 235 This work was supported by a NASA Office of the Chief Technologist's Space Technology Research Fellowship, and by NASA grant No. NNX13AF52G.

\section{References}

[1] L. L. Howell, Compliant Mechanisms, John Wiley Sons, Inc., 2001.

[2] C. Kim, D. Ebenstein, Curve decomposition for large deflection analysis of fixed-guided beams with application to statically balanced compliant 


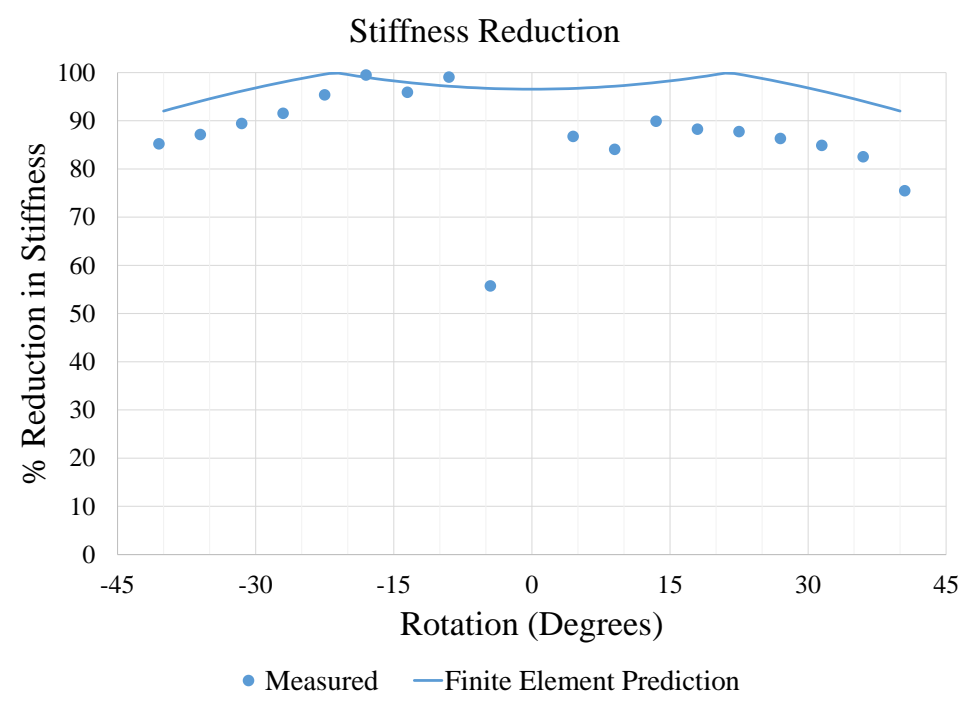

Figure 8: The percent reduction in stiffness of the balanced joint.

mechanisms, Journal of Mechanisms and Robotics 4 (4). doi:10.1115/1. 4007488

[3] J. L. Herder, Energy-free Systems. Theory, conception, and design of statically balanced spring mechanisms, Ponsen en Looijen BV, 2001.

[4] Design of spring force compensation systems, Mechanism and Machine Theory 33 (12) (1998) 151 - 161. doi:http://dx.doi.org/10.1016/ S0094-114X(97)00027-X.

[5] P. Pluimers, N. Tolou, B. D. Jensen, L. L. Howell, J. Herder, A compliant on/off connection mechanism for preloading statically balanced compliant mechanisms, DETC2012-71509, in: Proceedings of the ASME International Design Engineering Technical Conferences, ASME, 2012. doi: DETC2012-71509.

[6] A. Stapel, J. L. Herder, Feasibility study of a fully compliant statically balanced laparoscopic grasper, Vol. 2004, ASME, 2004, pp. 635-643.

[7] G. J. Tuijthof, J. L. Herder, Design, actuation and control of an anthropo- 
morphic robot arm, Mechanism and Machine Theory 35 (7) (2000) 945 962.

[8] N. Tolou, Statically balanced compliant mechanisms for mems and precision engineering, Dissertation (2012).

[9] J. Wang, C. Gosselin, Static balancing of spatial four-degree-of-freedom parallel mechanisms, Mechanism and Machine Theory 35 (4) (2000) 563592. doi : $\{10.1016 /$ S0094-114X (99) 00029-4\}.

[10] S. R. Deepak, G. Ananthasuresh, Static balancing of a four-bar linkage and its cognates, Mechanism and Machine Theory 48 (0) (2012) 62 - 80. doi:10.1016/j.mechmachtheory.2011.09.009.

[11] A compact low-stiffness six degrees of freedom compliant precision stage, Precision Engineering 37 (2) (2013) 380 - 388. doi:http://dx.doi.org/ 10.1016/j.precisioneng. 2012.10.007.

[12] D. J. de Lange, M. Langelaar, J. L. Herder, Towards the design of a statically balanced compliant laparoscopic grasper using topology optimization, in: ASME 2008 International Design Engineering Technical Conferences and Computers and Information in Engineering Conference, American Society of Mechanical Engineers, 2008, pp. 293-305.

[13] F. M. Morsch, J. L. Herder, Design of a generic zero stiffness compliant joint, in: ASME 2010 International Design Engineering Technical Conferences and Computers and Information in Engineering Conference, American Society of Mechanical Engineers, 2010, pp. 427-435.

[14] W. Wittrick, The properties of crossed flexure pivots, and the influence of the point at which the strips cross, Aeronautical Quarterly 2 (4) (1951) 272-292.

[15] B. R. Munson, D. F. Young, T. H. Okiishi, Fundamentals of Fluid Mechanics, John Wiley \& Sons, 2006. 
[16] J. Kennedy, R. Eberhart, et al., Particle swarm optimization, in: Proceedings of IEEE international conference on neural networks, Vol. 4, Perth, Australia, 1995, pp. 1942-1948.

[17] B. Jensen, L. Howell, The modeling of cross-axis flexural pivots, Mechanism and Machine theory 37 (5) (2002) 461-476.

[18] W. Wittrick, The theory of symmetrical crossed flexure pivots, Australian Journal of Scientific Research A Physical Sciences 1 (1948) 121.

[19] Z. Hongzhe, B. Shusheng, Accuracy characteristics of the generalized crossspring pivot, Mechanism and Machine Theory 45 (10) (2010) 1434-1448.

[20] H. Zhao, S. Bi, Stiffness and stress characteristics of the generalized crossspring pivot, Mechanism and Machine Theory 45 (3) (2010) 378-391. 